\section{International Scientific Journal Theoretical \& Applied Science}

\author{
p-ISSN: 2308-4944 (print) e-ISSN: 2409-0085 (online) \\ Year: $2018 \quad$ Issue: 02 Volume: 58
}

Published: $28.02 .2018 \quad$ http://T-Science.org

SECTION 21. Pedagogy. Psychology. Innovation in Education
Svetlana Anatol'evna Balyaeva Professor, Doktor of Pedagogical Science,

Professor Department of Physics, State Maritime University Admiral Ushakov,Russia, bs702@mail.ru

Tatyana Georgievna Khvingiya Associate Professor,

Assistant professor Department of Foreign Languages,

State Maritime University Admiral Ushakov, Russia, masalitinova@mail.ru

\title{
IMPLEMENTATION OF THE FOUNDATIONALIZATION PRINCIPLE IN THE PREPARATION PROCESS MARINE SPECIALISTS UNDER THE STCW CONVENTION REQUIREMENTS
}

\begin{abstract}
Present-day strategy of the fundamentalization of the educational process in the maritime university, pedagogical requirements for the design of basic and foreign language training of marine specialists based on the principle of fundamentalization; special attention is paid to the organization of educational and cognitive activities of students; the integrated training sessions, the third-line training are examined and their distinctive features are revealed; Increasing the efficiency of the educational process is associated with the use of innovative pedagogical technologies.

Key words: fundamentalization of the educational process, basic and foreign language training, experimental programs, integrated training sessions, training, electronic training tools.

Language: Russian

Citation: Balyaeva SA, Khvingiya TG (2018) IMPLEMENTATION OF THE FOUNDATIONALIZATION PRINCIPLE IN THE PREPARATION PROCESS MARINE SPECIALISTS UNDER THE STCW CONVENTION REQUIREMENTS. ISJ Theoretical \& Applied Science, 02 (58): 172-176.

Soi: http://s-o-i.org/1.1/TAS-02-58-36 Doi: crossef https://dx.doi.org/10.15863/TAS.2018.02.58.36

УДК 372.851

\section{РЕАЛИЗАЦИЯ ПРИНЦИПА ФУНДАМЕНТАЛИЗАЦИИ В ПРОЦЕССЕ ПОДГОТОВКИ МОРСКИХ СПЕЦИАЛИСТОВ В СООТВЕТСТВИИ С ТРЕБОВАНИЯМИ КОНВЕНЦИИ ПДНВ}

Аннотация: представлена современная стратегия фундаментализации образовательного процесса $в$ морском университете, выделены педагогические требования к проектированию базисной и иноязычной подготовки морских специалистов на основе принщипа фундаментализации; особое внимание уделено организации учебно-познавательной деятельности студентов; рассмотрены интегрированные учебные занятия, тренажерная подготовка и раскрыты их отличительные особенности; повышение эффективности образовательного прочесса связывается с использованием инновационных педагогических технологий.

Ключевые слова: фундаментализация образовательного процесса, базисная и иноязычная подготовка, экспериментальные программы, интегрированные учебные занятия, тренажерная подготовка, электронные обучающие средства.

\section{Introduction}

Российское высшее морское образование традиционно формировалось на принципах знаниевой парадигмы в соответствии с дидактической триадой «знания-умения-навыки ». Основное внимание уделялось усвоению знаний, так как считалось, что сам процесс их получения обладает развивающим потенциалом: именно в ходе такового формируются

необходимые умения и навыки ( теория развивающего обучения).

Современная стратегия высшего морского образования не перечеркивает прежнюю, а формируется на ее основе, выдвигая в центр внимания студента как активного субъекта, приобретающего образование не в процессе более или менее пассивного восприятия учебной информации, а в форме присвоения «личностного знания». Речь идет о принципиально новых целях
\end{abstract}


высшего морского образования, состоящих в достижении нового уровня образованности будущего специалиста морского транспорта. При этом «личностное знание» понимается как знание, делающее субъекта личностью; знание, которое в прямом смысле образует (творит) и образовывает (развивает) интеллект, личность [1].

Образование, разумеется, не существует вне знания. Но это должно быть знание определенного типа, происхождение которого уже невозможно установить, о нем не нужно вспоминать, оно полностью усвоено. Такое знание предполагает не «применение» законов, понятий и правил, а осмысление и понимание сути вещей и явлений. И в этом смысле можно считать, что «личностное знание» является фундаментальным знанием. Фундаментальные знания представляют собой стержневые, системообразующие, методологически значимые представления, восходящие к истокам понимания, к первичным сущностям.

Фундаментализация процесса подготовки морских специалистов означает его направленность на универсальные и обобщенные знания, на формирование общей культуры, развитие обобщенных способов деятельности, самого мышления. Это означает не увеличение объемов каждой из базисных, иноязычных и специальных дисциплин или сроков обучения, а переход к новым принципам отбора и систематизации знаний, создание инновационных учебных пособий, применение передовых педагогических технологий $[1,2,3]$.

\section{Materials and Methods}

На современном этапе развитие идеи фундаментализации образования обусловлено «интенсивным» подходом к формированию содержания учебных предметов - разработкой новых принципов построения учебных дисциплин и методов обучения, направленно формирующих мышление будущих специалистов и обеспечивающих повышение продуктивности познавательной деятельности обучаемых, их творческого саморазвития. В этой связи, в еще большей мере приобретает значение фундаментализация самих учебных дисциплин, и в первую очередь базисных и иноязычных, переход к новым, более обобщенным схемам теоретического описания их предмета, отражающих современный тип научного мышления, организация их усвоения в соответствующих видах познавательной деятельности .

С конструированием содержания учебных предметов тесно связана задача сделать содержание образования одновременно достаточно емким и доступным как по существу, так и по объему, привести его в соответствие с научно-техническими достижениями. Задачам фундаментализации содержания учебных предметов соответствует переход к новым схемам представления знаний о предмете, прежде всего, к методологическому описанию объектов в современной науке. Выделение этих схем, их усвоение как нормативов познавательной деятельности, раскрывающих фундамент предмета в новом ракурсе, формирование обобщенного типа ориентировки в любой учебной дисциплине составляют основу теоретического мышления специалиста нового типа. Эти схемы и должны стать новым более широким фундаментом воспроизводства научных знаний о предмете в процессе их усвоения, основанием их систематизации, принципом их функционирования в практической деятельности $[4,5,6]$

В качестве такой схемы при экспериментальном построении программы учебного предмета нами использовалась схема системного анализа. Системный принцип представления объектов содержанием учебного предмета, прокладывая схемы ориентации в предметной области, открывает студентам наличие глубокой упорядоченной связи между всеми объектами изучаемой действительности. Ориентация на эти связи формирует иные возможности - познавать явления окружающего мира на новом теоретическом уровне.

В соответствии с требованиями Международной конвенции о подготовке и дипломированию моряков и несении вахты (ПДНВ) нами разработаны на основе принципа фундаментализции экспериментальные программы по учебным дисциплинам базисного и иноязычного циклов подготовки в морском университете [7,8,9].

Эти программы построены таким образом, что вся система знаний о предмете базируется на логике системного анализа. Усвоение предметноспецифической системы знаний в единстве с методом раскрытия предмета изучения, как сложной системы, позволяет расширить возможности студентов по решению класса задач с учебно-профессиональным содержанием. отражающих типовые задачи будущей профессиональной деятельности.

$$
\text { Под учебными задачами с }
$$
профессиональным содержанием нами понимаются задачи прикладные, отражающие специфику будущей профессиональной деятельности студентов. В деятельности по их решению усваивается не только теоретический фундамент учебного предмета в его описании посредством категориального аппарата системного анализа, но и его прикладное 
содержание, выступающее при решении частных технических вопросов.

Специфическая особенность этих задач заключается в том, что они требуют выделения из описанной практической ситуации предметноспецифической задачи (установления из условий задачи сведений о реальном объекте, соотнесения его с соответствующей предметноспецифической моделью и выделения абстрактного объекта анализа, выявление всех его целостных свойств и характеристик, уяснение аспекта анализа выбранного объекта, т.е. собственно постановки задачи на анализ конкретного объекта с предметно-специфической точки зрения), разрешение поставленной задачи в рамках данной предметной области и интерпретации полученных результатов с предметной и профессиональной точек зрения, а в некоторых случаях и выдачи практических рекомендаций на основе проделанного анализа [10].

В соответствии с общей целью обучения предполагалось, что усвоение студентами предметно-специфических знаний в процессе деятельности по решению специально разработанного комплекса учебных заданий обеспечит возможность их использования не только как средства решения предметноспецифических задач, но и в их новой функции как средства формирования профессионального мировоззрения будущего специалиста. Эта новая функция базисных знаний и должна стать их ведущей функцией, как знаний «сущностных», фундаментальных. Заметим, что традиционное обучение не ориентировано на формирование этой функции базисных знаний.

Однако степень интегрирования базисных фундаментальных знаний и иноязычной компетенци, формируемых в процессе обучения в морском университете, в структуру общей профессиональной компетентности морских специалистов определяет уровень их готовности к эффективной и безопасной профессиональной деятельности [11].

Как известно, выпускник морского университета должен быть готов сразу после завершения образования приступить к практической работе в судовых условиях. Только фундаментальное образование может дать такие знания, которые позволяют ориентироваться в любой новой среде и являются универсальными по существу.

В свою очередь, фундаментализация и профессионализация содержания базисных учебных дисциплин и иноязычной подготовки дает возможность использования каждой дисциплины как методологического средства в дальнейшей учебной и профессиональной деятельности студента [2]. Формирование профессиональных компетенций будущих морских специалистов начинает осуществляться в процессе интеграции базисного и иноязычного образования, что способствует построению органически целостной системы профессиональной подготовки в морском университете, нацеленной на конечные результаты обучения, а именно, развитие навыков анализа и решения типовых профессиональных задач и умения оперативно действовать в реальных рабочих условиях, что, в свою очередь, помогает значительно сократить период адаптации молодого специалиста к его профессиональной деятельности $[12,13]$.

Одним из средств повышения эффективности интегрированной базисной и иноязычной подготовок морских специалистов является разработка дидактических комплексов обучения на базе квазипрофессиональных моделей с компьютерной поддержкой реальных технологических процессов труда на морском транспорте. При разработке данных дидактических комплексов учитываются такие важнейшие критерии как профессиональная значимость, профессиональная новизна, современность и актуальность. Отличительной особенностью этих комплексов является то, что они ориентированы на вовлечение студентов в выполнение практических заданий, связанных с применением получаемой информации в моделируемых квазипрофессиональных ситуация. При этом содержание профессионального труда представлено поэтапно в системе учебных проблем, задач и ситуаций, аналогичных профессиональным. В процессе учебной деятельности по выполнению этих заданий используется компьютерная поддержка, тренажерные программы и другие информационные ресурсы [14].

Важное место в совокупности педагогических условий, обеспечивающих формирование профессиональных компетенций студентов, занимают информационные и интерактивные педагогические технологии. Электронные образовательные средства, направленные на индивидуальную поисковую деятельность, способствуют тому, что студент не только закрепляет основные теоретические положения учебной дисциплины, но и учится самостоятельно выделять проблему, прогнозировать, планировать, выбирать оптимальный способ решения учебной ситуации, анализировать полученные результаты с предметно-специфической и там, где это необходимо, с квазипрофессиональной точек зрения, что в общем соответствует критериям профессиональной компетентности специалиста. 
Интерактивные формы учебных занятий предоставляют возможность формировать предметно-специфические, иноязычные и квазипрофессиональные знания и умения с достаточно высокой эффективностью при освоении базисного и иноязычного блоков профессиональной подготовки инженерноморских кадров через вариативный подход к решению типовых предметных заданий с профессионально ориентированным компонентом, создание ситуаций моделирования производственных функций, использование творческих заданий, деловых и позиционных игр с компьютерной поддержкой. Это обеспечивает интеграцию традиционных аудиторных занятий с инновационными формами их проведения.

\section{Conclusion}

Информационные технологии открывают широкие возможности для совершенствования преподавательской деятельности в процессе обучения и контроля, позволяя осуществлять индивидуальный подход к обучаемым в рамках лекционных и практических занятий, самостоятельно формировать поурочный учебный материал с учетом уровня подготовки студентов и оптимизировать соотношение объема учебного материала для аудиторных занятий и самостоятельной работы, а также быстро и эффективно осуществлять все виды контроля (текущий, промежуточный, итоговый) для проверки уровня усвоения учебного материала [15].

Неотъемлемой составной частью морского образования и основой безопасности мореплавания является тренажерная подготовка. Так, если при изучении цикла общенаучных и общеинженерных дисциплин от студента требуется усвоить основные базисные понятия, законы, определения, то в процессе обучения на тренажере он приобретает именно практические навыки эксплуатации и обслуживания различного судового оборудования. Такое сочетание двух разных подходов к морскому образованию, позволяет за счет взаимного дополнения и обогащения в значительной степени повысить уровень профессионализма в принятии решений операторами и командным составом судна, что, в конечном счете, оказывает огромное положительное влияние на снижение аварийности на судах.

В ходе деловых игр на тренажере отрабатываются комплексные задачи учебных дисциплин в контексте целостного технологического процесса в ситуациях иноязычного общения. Задания для ролевых и деловых игр, объединенные одной сюжетной линией (фрагмент несения вахты, отработка учений по тушению пожара и т.п.), постепенно усложняются и при спонтанном разыгрывании позволяют выявить уровень профессиональной компетенции студента в иноязычном общении [12].

Таким образом, тренажерная подготовка, наряду с интерактивными формами учебных занятий являются важными составляющими инновационной образовательной среды, формирующей практическую готовность студента к будущей профессиональной деятельности в морской отрасли.

В заключение заметим, что реализация принципа фундаментализации в образовательном процессе морского университета открывает новые пути повышения качества знаний выпускников, создает условия для эффективной интеграции базисной, иноязычной и специальной подготовки инженерно-морских кадров.

\section{References:}

1. Balyaeva S.A. (1999) Teoreticheskie osnovy' fundamentazizacii obshhenauchnoj podgotovki $\mathrm{v}$ sisteme vy'sshego texnicheskogo obrazovaniya . Avtoref. diss. dokt. ped. nauk.Moskva: Izd-vo MPGU.-1999. -32 p.

2. Mishhik S.A. (2016) Pedagogometricheskoe modelirovanie obrazovatel'noj deyatel'nosti // Uspexi sovremennoj nauki i obrazovaniya.Belgorod.2016.- № 8.- T. 1.- p. 85-87.

3. Uglova AN (2016) Design methodical maintenance of scientific disciplines
[Tekst]/A.N.Uglova // Materialy Mezhdunarodnoy nauchnoy konferenctsii «Global Science» - 30.04.2016 ISJ Theoretical \& Applied Science, 04 (36): 138 140. Lancaster, USA. http://dx.doi.org/10.15863/TAS.2016.04.36.22

4. (2002) Formirovanie sistemnogo my'shleniya v obuchenii / Pod red. prof. Z.A.Reshetovoj.- M.YuNITI-DANA.

5. Balyaeva SA (2016) Information model as a means of formation of professional integrity of 


\begin{tabular}{l|lr|ll|ll} 
& ISRA (India) & $=\mathbf{1 . 3 4 4}$ & SIS (USA) & $=\mathbf{0 . 9 1 2}$ & ICV (Poland) & $=\mathbf{6 . 6 3 0}$ \\
Impact Factor: & ISI (Dubai, UAE) $=\mathbf{0 . 8 2 9}$ & PUHЦ (Russia) $=\mathbf{0 . 2 0 7}$ & PIF (India) & $=\mathbf{1 . 9 4 0}$ \\
& GIF (Australia) & $\mathbf{0 . 5 6 4}$ & ESJI (KZ) & $=4.102$ & IBI (India) & $=\mathbf{4 . 2 6 0}$ \\
& JIF & $=\mathbf{1 . 5 0 0}$ & SJIF (Morocco) & $=\mathbf{2 . 0 3 1}$ & & \\
\hline
\end{tabular}

knowledge of maritime transport [Tekst] /S.A.Balyaeva // Materialy Mezhdunarodnoy nauchnoy konferenctsii «Global Science»30.04.2016 ISJ Theoretical \& Applied Science, 04 (36): 141-143. Lancaster, USA. http://dx.doi.org/10.15863/TAS.2016.04.36.23

6. Balyaeva SA, Uglova AN (2016) Innovative directions of the multi-level training program general scientific engineering and naval personnel [Tekst]/S.A.Balyaeva, A.N.Uglova// Materialy Mezhdunarodnoy nauchnoy konferenctsii «Technological advances» 30.03.2016 ISJ Theoretical \& Applied Science, 03 (35): 146-148. Philadelphia, USA. http://dx.doi.org/10.15863/TAS.2016.03.35.241

7. Balyaeva S.A. (2017) Didactic means of basic training of floating in the sea university Text./S.A.Balyaeva // Materialy Mezhdunarodnoy nauchnoy konferenctsii "Industry and technology" - 30.04.2017. ISJ Theoretical \&Applied Science 04 (48): 151-153 Philadelphia, USA. doi: https://dx.doi.org/10.15863/TAS.2017.04.48.24

8. Balyaeva S.A. (2017) Kompleksny'j podxod k modernizacii obrazovatel'nogo processa na e'tape bazovoj podgotovki specialistov morskogo flota / Obrazovanie i nauka: sovremenny'e trendy': kollektivnaya monografiya / gl. red. O.N.Shirokov Cheboksary': CNS «Interaktiv plyus », 2017.p. 90-101.

9. Tokmazov G.V., Balyaeva S.A., Pan'kina S.I. (2017) Konkretizaciya osnovny'x urovnej podgotovki pri modernizacii matematicheskogo obrazovaniya studentov // Uspexi sovremennoj nauki i obrazovaniya. - 2017.- № 5.- p. 197201.

10. Balyaeva S.A., Uglova A.N. (2012) Povy'shenie e'ffektivnosti obshhenauchnoj podgotovki specialistov morskoj otrasli na baze innovacionny'x didakticheskix texnologij // Nauchny'e problemy' gumanitarny'x issledovanij. Nauchno-teoreticheskij zhurnal.Pyatigorsk, 2012.- № 5.- p. 95-106.

11. Balyaeva S.A. (2017) Pedagogical innovations in the context of forming the professional competence of engineering specialists of the marine branch Text./S.A.Balyaeva // Materialy Mezhdunarodnoy nauchnoy konferenctsii "Technological breakthrough in science" 30.03.2017. ISJ Theoretical \&Applied Science 03 (47): 139-142 Philadelphia, USA. doi: https://dx.doi.org/10.15863/TAS.2017.03.47.24

12. Balyaeva S.A. Xvingiya T.G. (2017) Aktual'ny'e problemy' innovacionnogo obespecheniya integrirovannoj bazovoj i inoyazy'chnoj podgotovki plavsostava $\mathrm{v}$ morskom universitete: Nauchny'e issledovaniya - 2017: prakticheskaya chast'. Monografiya [E'lektronny'j resurs]. - M.: RusAl'yans Sova. 2017. - Vy'pusk 3. -p. 82-94

13. Balyaeva S.A., Uglova A.N. (2014) Innovacionny'e didakticheskie texnologii kak sredstvo formirovaniya universal'ny'x professional'ny' $x$ kompetencij inzhenerov morskogo flota / V kn.: Obrazovatel'noinnovacionny'e texnologii: teoriya i praktika: monografiya / Pod obshhej red. prof. O.I.Kirikova.- Kniga 19 - Moskva: Nauka: inform; Voronezh: VGPU, 2014. -p. 121-135.

14. Balyaeva, S.A. Xvingiya T.G. (2017) E'lektronny'e obuchayushhie sredstva kak rezerv optimizacii uchebnogo processa $\mathrm{v}$ morskom universitete // Izvestiya Yuzhnogo federal'nogo universiteta. Pedagogicheskie nauki. 2017.- № 5.- p.65-75.

15. Balyaeva S.A., Baloyan O.N. (2014) Puti optimizacii kontrolya obshhenauchnoj podgotovki $\mathrm{v}$ sfere vy'sshego morskogo obrazovaniya // Izvestiya Yuzhnogo federal'nogo universiteta. Pedagogicheskie nauki. 2014.- № 4.- p.93-100. 